\title{
Research on Multi-physical Modeling and Co-simulation of Aircraft
}

\author{
Dangdang Zheng, Liqiang Ren, Ying Wu, and Juntang Liu
}

\begin{abstract}
Advanced aircraft system is more and more integrated, which makes the problem of multiple physical coupling between different physical systems more and more complex. Multi-physical modeling and co-simulation is a kind of important means for the early design study of multi-physical coupling, supporting systems integrated design and verification. Combined with the methodology of model-based system engineering, the key technologies of multi-physical system modeling and co-simulation are systematically analyzed, and the engineering application mode of multi-physical co-simulation for different stages of aircraft development is proposed.
\end{abstract}

D. Zheng $(\bowtie)$

Northwestern Polytechnical University, Xi'an, People's Republic of China

e-mail: zhengdangdang@126.com

D. Zheng $\cdot$ L. Ren $\cdot$ Y. Wu $\cdot$ J. Liu

The First Aircraft Institute of AVIC, Xi' an, People's Republic of China

e-mail: 18717393589@163.com

Y. Wu

e-mail: wuying@163.com

J. Liu

e-mail: juntangliu@163.com 